PAGINA DE OUTROS PROFISSIONAIS

\title{
PERIODICOS PARA O CURSO DE GRADUAÇÃO EM ENFERMAGEM E OBSTETRÍCIA NO BRASIL: ESTUDO BIBLIOMÉTRICO, 1966-1976*
}

\author{
Carmen Sylvia Arantes Leal Aguiari**
}

$\operatorname{ReBEn} / 10$

AGUIARI, C.S.A.L. - Periódicos para o Curso da Graduação em Enfermagem e Obstetrícia no Brasil: Estudo Bibliométrico, 1966-1976. Rev. Bras. Enf.; DF, 34 : 333-342, 1981.

Conhecendo a eficácia das listas básicas de periódicos que foram elaboradas em outras áreas, com a finalidade de facilitar a atualização dos conhecimentos dos profissionais e contribuir para a racionalização das verbas e do espaço nas bibliotecas; e, dada a inexistência de uma lista básica de periódicos em Enfermagem e Obstetricia, resolvemos fazer este estudo, como uma contribuição às bibliotecas desta área, para a difícil tarefa de seleção dos periódicos.

\section{INTRODUÇAO}

O periódico científico é inquestionavelmente o material bibliográfico mais importante no acervo de uma biblioteca universitária dada a sua contribuição efetiva tanto para o ensino como para a pesquisa.

Constitui um veículo de comunicação eficiente entre os profissionais nas diver'sas áreas do conhecim'ento humano, pois permite que o fluxo internacional de idéias $\theta$ informações se faça de maneira rápida, freqüente e constante.

Entretanto, a sempre crescente quantidade e variedade de títulos editados resulta em séries dificuldades para os estudiosos, que precisam despender tempo cada vez maior a fim de selecionar dentre a massa de artigos publicados, os novos conhecimentos $\mathbf{e}$ idéias de que necessitam para se manterem atualizados.

* Resumo baseado na Dissertação apresentada à Escola de Comunicação e Artes da USP em 29/8/1980, para obtenção do grau de mestre em Ciência da Comunicação.

** Bibliotecária-Chefe da Biblioteca da Escola de Enfermagem da USP. 
AGUIARI, C.S.A.L. - Periódicos para o Curso da Graduação em Enfermagem e Obstetrícia no Brasil: Estudo Bibliométrico, 1966-1976. Rev. Bras. Enf.; DF, 34 : 333-342, 1981.

Este fato dificulta extremamente ao estudioso acompanhar a literatura de seu interesse, o que exige a existência de bibliografias específicas ou outros instrumentos auxiliares que possibilitem consulta imediata.

Outra conseqüência desse exagero de publicações periódicas é o problema que representa, para as bibliotecas universitárias, sua seleção, aquisição e espaço para as coleções.

Os processos de seleção, aquisição e previsão de espaço para as coleções, por sua vez exigem conhecimento de particularidades pertinentes a cada uma delas.

Além desses aspectos, devemos considerar os problemas de ordem técnica e administrativa, entre os quais: previsão orçamentária, registro, tombamento, catalogação, preparo técnico, descarte e encadernação.

Por todos esses motivos, os periódicos devem ser selecionados criteriosamente e adquiridos mediante normas que possibilitem o rendimento máximo dos recursos financeiros existentes, sempre deficientes.

Na revisão bibliográfica pertinente à área da saúde, espesificamente a da Enfermagem, identificamos diversos autores que realizam estudos de certa maneira afins ao nosso, dos quais citamos alguns: STEARNS 20, 21, BRANDON ${ }^{3}$, DURANA 9, CONCÓRDIA 7, KARAN 14.

Na revisão bibliográfica referente à Biblicteconomia e Documentação, destacamos certos trabalhos que estabeleceram bases sólidas desta área, entre eles: OTLET 16, ZOLTOWSKI 23 e BRADFORD $^{2}$ que criou a metodologia conhecida como a lei de Bradford.

$\mathrm{Na}$ ciência da informação, a lei de Bradford faz parte da "Bibliometria", definida como a área do conhecimento que estuda os métodos para o "tratamento quantitativo do discurso gravado e o procedimento a ser seguido" 10 .
No Brasil, foram aplicadas as leis bibliométricas nas seguintes áreas: física 12, botânica 17, biologia 6 , geologia 11, microbiologia-imunologia-parasitclogia 19 , saúde pública 1 , e em ciências agrícolas 18 .

Na falta de um método eficaz de seleção de periódicos que atenda aos interesses do ensino e da pesquisa em Enfer'magem e Obstetrícia ${ }^{8},{ }^{15}$ escolhemos a metodologia de Bradford, que é a única existente, para elaborar uma lista básica nesta área.

OBJErIVOS: o presents estudo foi realizado com a finalidade de alcançar os seguintes objetivos:

1 - identificar a lista básica de periódicos necessários do curso de graduação em Emfermagem e Obstetrícia no Brasil;

2 - relacionar os periódicos que tratam com maior freqüência dos assuntos pertinentes às matérias do currículo mínimo do curso de graduação em Enfermagem e Obstetrícia no Brasil;

3 - quantificar a produção nesta área do conhecimento.

\section{METODOLOGIA}

Os dados para coleta retirados da cbra de referência "INTERNATIONAL NURSING INDEX" 13 (I.N.I. que é uma publicação com as seguintes características: bibliografia especializada, corrente e de âmbito internacional.

UNIVERSO - O universo estudado foram todos os artigos publicados nos periódicos científicos da área em pauta e indexados no I.N.I. 13. Esta publicação é sinalética, isto é, enuncia apenas as referências bibliográficas dos artigos; pois nesta área não existe publicação analítica (referência acompanhada de resumo). E publicada trimestralmente, sendo os fascículos reunidos e reedita- 
AGUIARI, C.S.A.L. - Periódicos para o Curso da Graduação em Enfermagem e Obstetrícia no Brasil: Estudo Bibliométrico, 1966-1976. Rev. Bras. Enf.; DF, 34 : 333-342, 1981.

dos em volume cumulativo, ao término de cada ano. O I.N.I. foi iniciado em 1966 com a publicação do volume 1, que indexou 161 periódicos em Enfermagem; no volumie 11 , de 1976, foram indexados 296 periódicos específicos de Enfermagem, além de outros na área da saúde. A parte mais importante a ressaltar no I.N.I. é a SEÇÃO de ASSUNTOS, ordenada alfabeticamente; sob cada cabeçalho aparecem as referências bibliográficas de periódicos, folhetos e partes de livros que tratam do assunto.

Para o conhecimento das matérias estudadas recorremos à Resolução n..$^{4}$, de 25/2/1972, do Conselho Federal de Educação 4, (C.F.E.) baseada no Parecer n. ${ }^{\circ} 163 / 72$ do mesmo Conselho, que fixa o currículo mínimo do curso de graduação. Este compreende três partes sucessivas:

\section{a) pré-profissional;}

b) tronco profissional comum;

c) habilitação.

Para este estudo, foi selecionada a parte "b", referente ao "tronco profissional comum", que é a fração de maior duração do curso, que abrange, segundo o Parecer n. ${ }^{\circ}$ 163/72, do C.F.E. as seguintes matérias;

$$
\begin{aligned}
\text { I - Introlução à Emfermagem; } \\
\text { II - Enfermagem Médico-Cirúrgica; } \\
\text { III - Enfermagem Materno-Infantil; } \\
\text { IV - Enfermagem Psiquiátrica; } \\
\text { V - Enfermagem em Doenças Trans- } \\
\text { missíveis; } \\
\text { VI - Exercício da Enfermagem, in- } \\
\text { cluindo Deontologia Médica e } \\
\text { Legislação Profissional; } \\
\text { VII - } \\
\text { Didática aplicada à Enferma- } \\
\text { gem; } \\
\text { VIII - } \\
\text { Administração aplicada à En- } \\
\text { fermagem. }
\end{aligned}
$$

Para preencher as matérias acima, selecionamos as rubricas do I.N.I. em inglês e em português, que resultam dos assuntos abrangentes nas disciplinas as quais relacionamos a seguir:

$\begin{array}{ll}\text { I - Philosophy, Nursing } & \begin{array}{l}\text { Filosofia de Enfermagem } \\ \text { Nursing care } \\ \text { Diagnosis } \\ \text { Techniques and Procedures, see } \\ \text { Nursing care; specific procedu- } \\ \text { res subheading "methods" }\end{array} \\ \begin{array}{ll}\text { Diagnóstico } \\ \text { Técnicos e procedimentos }\end{array} \\ \text { II Cardiovascular Diseases } & \\ \begin{array}{l}\text { Diabetes Mellitus } \\ \text { Diabetes Mellitus Juvenile }\end{array} & \text { Doenças cardiovasculares } \\ \text { Geriatric Nursing } & \text { Diabetes M. } \\ \text { Kidney Diseases } & \text { Diabetes J. } \\ \text { Neurology } & \text { Enfermagem geriátrica } \\ \text { Rheumatism } & \text { Doenças renais } \\ \text { Rheumatism fever } & \text { Neurologia } \\ \text { Rheumatic heart Disease } & \\ \text { Thoraxic Diseases } & \text { Febre reumática } \\ \text { Diagnosis, Laboratory } & \text { Doença cardiaca reumática } \\ \text { Surgical Nursing } & \text { Doenças toráxicas } \\ \text { Preoperative care } & \text { Diagnóstico de laboratório } \\ \text { Postoperative care } & \text { Enfermagem cirúrgica } \\ & \text { Cuidados pré-operatórios }\end{array}$


AGUIARI, C.S.A.L. - Periódicos para o Curso da Graduação em Enfermagem e Obstetrícia no Brasil: Estudo Bibliométrico, 1966-1976. Rev. Bras. Enf.; DF, 34 : 333-342, 1981.

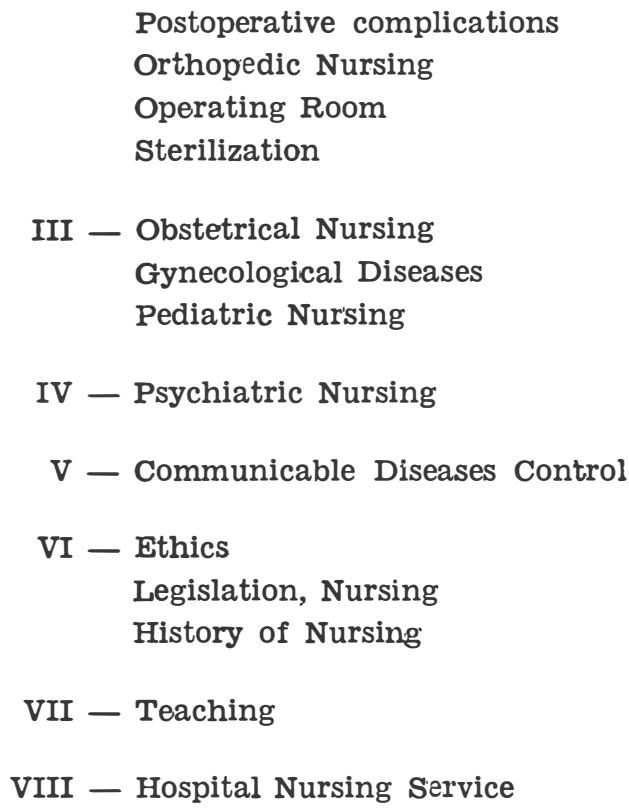

Postoperative complications Orthopedic Nursing

Operating Room

Sterilization

III - Obstetrical Nursing

Gynecological Diseases

Pediatric Nur'sing

IV - Psychiatric Nursing

V - Communicable Diseases Control

VI - Ethics

Legislation, Nursing

History of Nursing

VII - Teaching

VIII - Hospital Nursing Service

Complicações pós-operatórios

Enfermagem crtopédica

Sala de Operação

Esterilização

Enfermagem obstétrica

Doenças ginecológicas

Enfermagem pediátrica

Enfermagem psiquiátrica

Controle de doenças transmissíveis

Etica

Legislação da Enfermagem

História da Enfermagem

Pedagogia e Didática

Serviços de Enfermagem hospitalar

Adicionamos no estudo 4 disciplinas, a saber: Farmacologia e Dietoterapia freqüentemente ministradas simultaneamente àquelas; Enfermagem em Saúde Pública e Metodologia Científica, que sabemos constar do currículo do tronco profissional comum em algumas escolas 22.

\author{
Nutrition \\ Diet Theraphy \\ Child Nutrition \\ Drugs administration \\ Immunization \\ Preventive Health care \\ Health Services \\ Community Health services \\ Research
}

PERIODO: O presen'te estudo abrange o periodo correspondente aos anos de 1966 a 1976, inclusive, do I.N.I.

A coleta de dados foi feita no período de janeiro de 1977 a julho de 1978 .

Num estudo piloto, foram levantados os dados correspondentes aos anos de 1966 a 1967, coincidentes com os volumes 1 e 2 do I.N.I.; apesar de restri-

\author{
Nutrição \\ Dietoterapia \\ Nutrição infantil \\ Administração e dosagem de dro- \\ gas \\ Imunização \\ Cuidados preventivos de saúde \\ Serviços de Saúde \\ Serviços comunitários de saúde \\ Pesquisa
}

to, trouxe subsídios para o presente estudo.

INSTRUMENTO: O instrumento de pesquisa para o levantamento dos dados foi uma ficha, contendo:

- cabeçalho de identificação do pe-
riódico e do assunto; 
AGUIARI, C.S.A.L. - Periódicos para o Curso da Graduação em Enfermagem e Obstetricia no Brasil: Estudo Bibliométrico, 1966-1976. Rev. Bras. Enf.; DF, 34 : 333-342, 1981.

- coluna indicativa da esquerda; meses do ano, e local para somatória;

- linha horizontal superior, os anos de 1966 a 1976, e local para somatória.

Usamos uma ficha individual para cada título de periódico que abordasse cada assunto pesquisado.

COLETA DE DADOS: Na seção de assuntcs do I.N.I., procuramos o cabeçalho correspondente às matérias já relacionadas em material. Sob cada cabeçalho, encontramos a relação de referências bibliográficas que tratava do assunto em pauta. Dessas, selecionamos somente as referências bibliográficas de periódicos $e$ os seguintes elementos: título do periódico, mês e ano. Ano'tamos em cada ficha o núməro de artigos (frequiência) sobre o assunto publicado no reriódico mencionado no cabeçalho.

APURAÇÃO dos DADOS: Em cada ficha, efetuamos a soma das linhas e das colunas e anotamos os totais no local para somatória, na mesma ficha. Deste modo, apuramos os dados, e reordenamos as fichas desta vez em ordem dz produtividade decrescente de cada assunto analisado.

BIBLIOMETRIA: Bradford, formulou sua lei de literatura periódica nos seguintes termos: "Se os periódicos forem organizados em ordem de produtividade decrescente de artigos pertinen'tes a um determinado assunto, estes periódicos poderão ser divididos em um núcleo mais particularmente dedicado ao assunto e em diversos grupos ou zonas contendo o mesmo número de artigos que o núclieo, enquanto que o número de periódicos existentes no núcleo e nas zcnas subseqüentes será da ordem de $1: n: n^{2} \ldots{ }^{5}$.
TABELAS E GRÁFICOS: Os dados apurados em cada assunto foram transportados para as tabelas, e, logo após, foram novamente transportados para os gráficos. Nos gráficos foram traçados linhas de união entre os pontos, os quais apontam um núcleo, som um certo número de periódicos mais dedicados a cada assunto analisado.

Em atenção ao enunciado da lei de Bradford, selecionamos para este estudo somente o núcleo apontado em cada assunto pesquisado.

A partir dos núcleos, podemos identificar quais os periódicos mais prołutivos em cada um dos assun'tos analisados.

Com todos os dados da pesquisa, pudemos então elaborar uma Tabelamestra que aponta todos os resultados obtidos. Esta contém na primeira coluna à esquerda, em ordem alfabética, os periódicos apontados nos núcleos dos gráficos; e na horizontal, os 37 (trinta e sete) assuntos pesquisados, em ordem decrescente de produtividade (freqüência).

O procedimento por nós adotado para a'tingir o primeiro objetivo foi a seleção dos periódicos que abordaram maior quantidade de assuntos de acordo com a Tabela em anexo. Com o auxílio de um esquadro, anotávamos à parte o título do periódico, bem como os assuntos por eles abordados; deste modo identificamos a lista básica.

Quanto ao segundo objetivo, utilizamos a mesma tabela, com o auxilio de uma régua para as colunas, anotávamos à parte o assunto pesquisado, bem como os títulos dos periódicos, número de artigos publicados em cada um deles, em ordem decrescente de produtividade.

Quanto ao terceiro objetivo, recorremos novamente à tabela e anotamos os dados à parte: o assunto, número de artigos em cada um deles, e o total de periódicos que abordavam o mesmo; 
AGUIARI, C.S.A.L. - Periódicos para o Curso da Graduação em Enfermagem e Obstetrícia no Brasil: Estudo Bibliométrico, 1966-1976. Rev. Bras. Enf.; DF, 34 : 333-342, 1981.

deste modo pudemos quantificar a produção de artigos.

\section{RESULTADOS}

Sobre os trinta e sete assuntos pesquisados foram identificados 18.233 artigos científicos indexados no I.N.I., contidos em 3.724 periódicos, de $1 .^{\circ}$ de janeiro de 1966 a 31 de dezembro de 1976.

A distribuição dos artigos indexados nos periódicos, segundo os assuntos pesquisados, foi disposta em ordem decrescente de produtividade. A tabela-mestra engloba todo o resultado de nossa pesquisa, relacionamos na linha horizontal os dados referentes ao universo da pesquisa, numeradas de 1 a 37. Abaixo de cada um é apresentada a quantificação dcs artigos, em ordem decrescente de produtividade, cuja freqüência varia de 2.649 a 47, e o número total de periódicos, em cada assunto analisado, com a freqüência que varia de 331 a 21.

A miesma tabela agrupa, na coluna à esquerda, os 78 periódicos apontados nos núcieos dos gráficos referentes a cada assunto analisado, o que permite identificar o número de artigos neles publicados. Tais periódicos são apresentados em ordem alfabética. Pode-se verificar, na linha inferior de total: 1) - número total de artigos nos núcleos dos assuntos analisados e a freqüência que varia de 1.115 a $18 ; 2$ ) número de periódicos em cada núsleo e a freqüência que varia de 21 a 3 ; na coluna de total à direita: 1) - número de artigos agrupados por periódicos e a freqüência que varia de 1.344 a 3 ; 2) _número de assuntos abrangidos em cada periódico e a frequência que varia de 29 a 1.

\section{DISCUSSĀO}

Quanto ao material: a utilização de bibliografia in'ternacional corrente, como material de coleta de dados, seria a diretriz mais eficiente, pela abertura de assuntos que poderiam ser analisados. Mas, este motivo apontou inúmeros periódicos em língua pouco conhecida pela maioria dos brasileiros; por outro lado, nos deu uma noção da produção intelectual neste campo em outros paises

Quanto ao método: a aplicação da metodologia de Bradford aponta uma concentração estátística de títulos de periódicos, não é considerado o valor informativo das fontes, uma vez que a lei não emite conceitos quanto à qualidade dos artigos publicados. No entanto, a utilização da lei de Bradford constitui um instrumento seguro para a obtenção por nós desejada para atingir os três objetivos inicialmente propostos por este estudo.

Quanto aos resultados: idioma dos títulos listados na coluna à esquerda da tabela, perfazem um total de 78, dos quais 4 mudam de título, e um deixou de ser publicado Ficaram, pois, 73 títulos, dos quais 13 são periódicos que publicam artigos na área da saúde Os 60 restan'tes são específicos de enfermagem. Deste total de 60 periódicos, 34 são em inglês, 9 em japonês, 2 em russo e 2 em tcheco-eslovaco Os 13 restantes são em outras línguas, nenhum 'em português.

Periodicidade das publicações: American Journal of Nursing (Now York) iniciado em 1900, sem interrupção, é uma publicação mensal Nursing Times (Inglaterra) iniciado em 1905, sem interrupção, é semanal e o Nursing Mirror and Midwives Journal (Inglaterra) iniciado em 1888, sem interrupção, é semanal.

Número de artigos: em determinados assuntos, consta do núcleo grande número d'e artigos e sobre outros, que consideramos importantes e que supúnhamos atraírem a atenção dos autores, o número de artigos é restrito Ex.: Serviços de Enfermagem Hospitalar, 2.649 
AGUIARI, C.S.A.L. - Periódicos para o Curso da Graduação em Enfermagem e Obstetricia no Brasil: Estudo Bibliométrico, 1966-1976. Rev. Bras. Enf.; DF, 34 : 333-342, 1981.

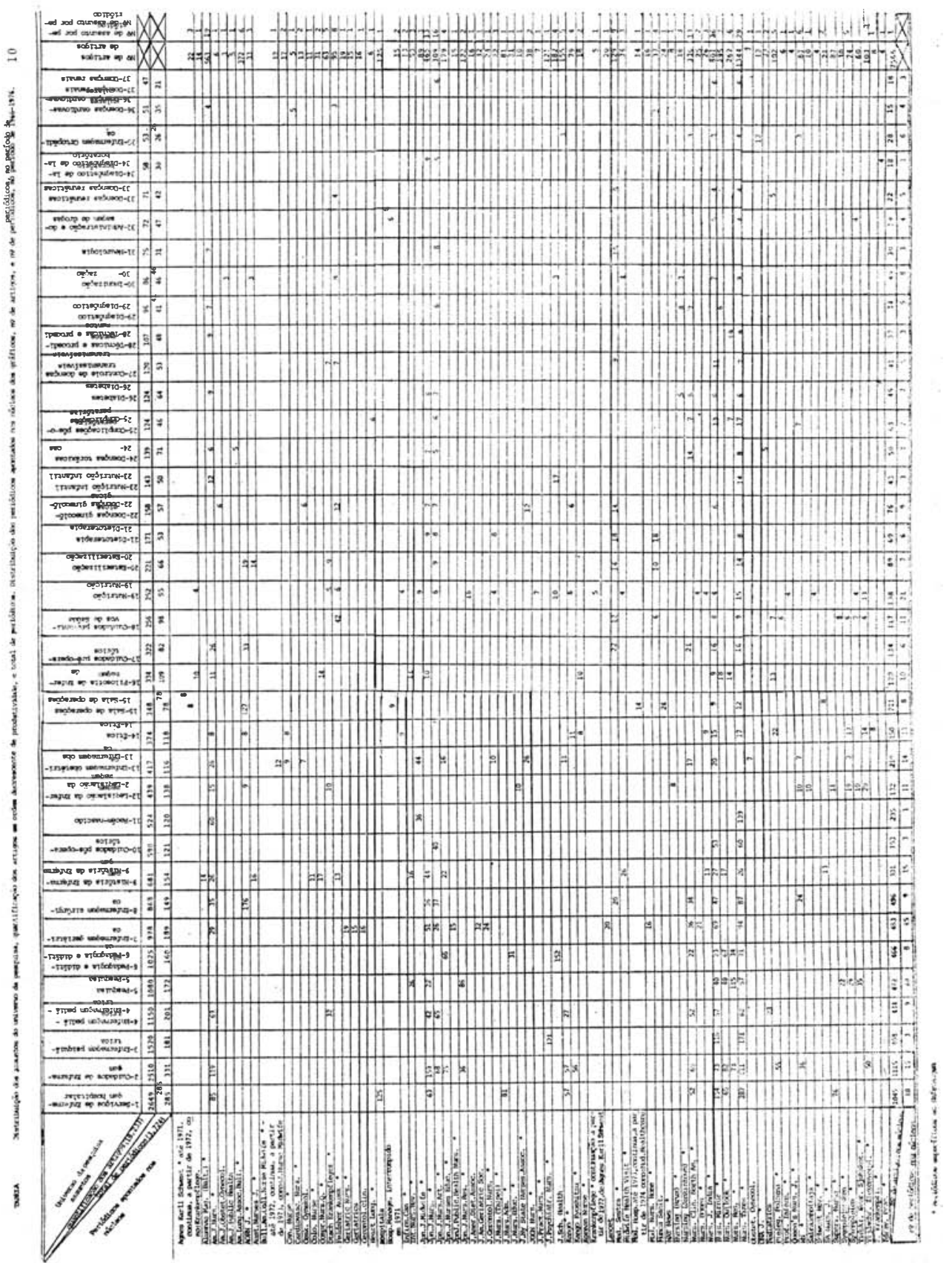


AGUIARI, C.S.A.L. - Periódicos para o Curso da Graduação em Enfermagem e Obstetrícia no Brasil: Estudo Bibliométrico, 1966-1976. Rev. Bras. Enf.; DF, 34 : 333-342, 1981.

artigos e Doenças cardiovasculares, 51 artigos, no periódico de 11 anos.

Fizemos o estudo com a finalidade de selecionar alguns dos muitos problemas relacionados com os periódicos científicos, para atender o aspecto administrativo dos mesmos.

Para isso, utilizamos a metodologia e obtivemos resultados que nos forneceram subsídios ao aspecto quantitativo.

Ressalvamos que, conscientemente, não apontamos listas $\Theta$ para tal solicitamos o apoio de uma comissão de profissionais nesta área de estudo, para avaliar os periódicos, quanto à profundidade dos termos abordados, quanto ao corpo editorial, para que possamos atingir o aspecto qualitativo e ser válida nossa proposta. Finalmente, com esse estudo esperamos auxiliar os profissionais responsáveis pelo estudo.

\section{CONCLUSŌES}

Identificar a lista básica de periódicos necessários aos cursos de graduação em Enfermagem e Obstetrícia no Brasil, a qual ficou assim constituída: NURSING TIMES: além de abranger 29 assuntos, é dedicado aos seguintes: 1, 2, 3, $4,5,6,7,8,9,10,11,14,15,17,18,19$, $20,21,23,24,25,26,27,28,30,32,35$ e 37 (relacionados em linha horizontal da tabela). NURSING MIRROR and MIDWIVES JOURNAL: além de abranger 26 assuntos, é dedicado aos seguintes: 13,16 e 22 (relacionados em linha horizontal da tabela). THE AMERICAN JOURNAL of NURSING: além de abranger 19 assuntos, é dedicado aos seguintes: $12,29,31$ e 36 (relacionados em linha horizontal da tabela).

A lista de títulos de periódicos mais adequados a cada um dos assuntos pértinentes às matérias do curso de graduação em Enfermagem e Obstetrícia no Brasil: com o auxílio de uma régua apontada para os assuntos de interesse, localizamos os periódicos em ordem de- crescente da produtividade, bem como na coluna à esquerda, o título do periódico correspondente ao número de artigos nele incluídos. Quantificar a produção intelectual indexada no I.N.I. referente aos assun'tos pesquisados. Novamente utilizamos a tabela e, na linha inferior de total, o número de artigos em cada assunto analisado.

\section{SUGESTŌES}

Com base em nossa experiência de 14 anos de chefia de biblioteca, e nos resultados do presente estudo, ousamos apresentar algumas sugestões. Como pode ser observado na tabela, não há periódicos em língua portuguesa. Seus artigos sobre cada assunto, embora indexados no I.N.I., não são em número suficiente para figurar no núcleo. A razão é simples, os dois periódicos ingleses são semanais, o mericano é mensal, mais volumoso e cada fascículo contém grande número de artigos.

- Que as revistas de enfermagem publicadas no Brasil sejam incluídas na lista básica de periódicos, entre elas temos:

REVISTA BRASILEIRA de ENFERMAGEM, órgão oficial da Associação Brasileira de Enfermagem. E trimestral.

REVISTA da ESCOLA de ENFERMAGEM da USP, publicada desde 1967. quadrimestral.

ENFERMAGEM em NOVAS DIMENSÕES, publicação suspensa em 1979. Esperamos que volte em breve a circular.

As publicações acima são editadas em língua portuguesa, e de fácil aquisição, além disso o seu custo é relativamente baixo.

- Que se dêem às bibliotecas condições de elaborarem previsão orçamentária apropriada para utilização eficaz dos recursos disponiveis, permitindolhes adquirir os periódicos apontados na lista básica. 
AGUIARI, C.S.A.L. - Periódicos para o Curso da Graduação em Enfermagem e Obstetrícia no Brasil: Estudo Bibliométrico, 1966-1976. Rev. Bras. Enf.; DF, 34 : 333-342, 1981.

- Que, havendo verba suficiente, se estenda a aquisição dos periódicos relacionados aos assuntos analisados, por constituírem instrumentos de apoio aos estudiosos.

- Que as bibliotecas possam dispor do espaço necessário às coleções adquiridas.

- Que os resultados deste estudo ajudem os serviços de informação especializados na seleção dos periódicos a serem prioritariamente abrangidos na elaboração de bibliografias analíticas, inexistentes na área estudada.

- Que os editores das revistas brasileiras de Enfermagem modifiquem a programação da periodicidade dessas publicações dando-lhes freqüência maior, mensal, quinzenal ou semanal, o que viria contribuir para a indexação de maior número de artigos em cada assunto.

- Que cs autores e colaboradores das publicações brasileiras, ao elaborarem suas experıencias profissionais, obedeçam aos padrões nacionais e internacionais quanto à extensão dos artigos, qu'e deverão conter no mínimo uma lauda e meia e, no máximo, três laudas e meia.

Este trabalho inicial cobriu apenas o tronco profissional comum do currículo de graduação. Seria conveniente que fosse feita também lista básica de periódicos para os períodos pré-profissional e de habilitação, bem como para pós-graduação.

\section{B I B L I O G R A F I A}

1. ANDRADE, M. T. D. de et alii. Avaliação do uso de periódicos em biblioteca especializada em saúcie pública. Rev. Saúde pública, São Paulo, $12(3): 388-402,1978$.

2. BRADFORD, S. C. O caos documentário. In: ........ Documentação. São Paulo, Fundo de Cultura, 1961, cap. 9, págs. 198-216.

3. BRANDON, A. N. Selected list of books and journals for the small medical library. Bull. med. Libr. Ass. Chicago, 59(2):266-85, Apr. 1971.

4. BRASIL. Ministério da Saúde. Fundação Serviços de Saúde Pública. Resolução n. ${ }^{2}$ 21/72, de 25.2.1972, dis........

5. BROOKES, B. C. Bradford's law and the bibliography of science. Nature, London, 224:953-6, Dec. 1969.

6. CARVAlHo, M. L. B. de. Estudo de citações da literatura produzida pelos professores do Instituto de Ciências Biológicas da UFMG. Ci. Inf., Rio de Janeiro, 5(1/2):27-42, 1976.

7. CONCORDIA, M. Sister. Basic book and periodical list: nursing school and small medical library. 4. ed. Illinois, st. Bede Abdey Press, 1967. 144 págs.

8. DISSERTATION ABSTRACTS INTERNATIONAL. Section A: the humanites and social sciences: library science. Michigan, Xerox University Microfilms, 1971-1977. v. 31-38.

9. DURANA, I. Selected library references in spanish for basic nursing program in Latin America. Colombia, 1966. (Dissertation for the degree of Master of Science in Nursing The University of Valle Schcol of Nursing - Cali).

10. FAIRTHORNE, R. A. Empirical hyperbolic distributions (Bradford-ZipfMandelbrot) for bibliometric description and prediction. J. Documentation, London, 25(4):319-43, Dec. 1969.

11. FIGUEIREDO, L. M. Distribuição da literatura geológica brasileira: estudo bibliométrico. Ci. Inf., Rio de Janeiro, 2(1):27-40, 1973.

12. GOMES, T. F. \& MARQUES, S. A. Seleção de periódicos científicos para a área da física. Ci. Inf., Rio de Janeiro, 3(2) :105-46, 1974.

13. INTERNATIONAL NURSING INDEX annual cumulation. New York, American Journal of Nursing, 1366-1976. v. $1-11$.

14. KARAN, A. M. S. et alii. Requisitos minimos para a organização e funcionamento das bibliotecas das escolas de enfermagem. São Paulo, BIREME, 1973. 43 págs. (Mimeografado). 
AGUIARI, C.S.A.L. - Periódicos para o Curso da Graduação em Enfermagem e Obstetrícia no Brasil: Estudo Bibliométrico, 1966-1976. Rev. Bras. Enf.; DF, 34 : 333-342, 1981.

15. MASTERS ABSTRACTS. Michigan, Xerox University Microfilms, 1973, v. 11 .

16. OTLET. P. Le livre et la mesure: bibliométrie. In: ........ Traité de documentation: le livre sur le livre; théorie et pratique. Bruxelas, $\mathrm{Pa}$ lais Mondial, 1934, págs. 13-22

17. QUEIROZ, S. de S. Bibliografia brasileira de botânica, 1971-1972: estudo bibliométrico. Ci. Inf., Rio de Janeiro, 4(1):55-66, 1975.

18. ROBREDO, J. et alii. Metodologia para a elaboração de lista básica dos periódicos nacionais em ciências agrícolas e estudo da dispersão da literatura agrícola brasileira. $R$. Bibliotecon. Brasília, Brasília, 2(2): 119-42, jul./dez. 1974.

19. SA, E. S. Participação dos pesquisadores de microbiologia, imunologia e parasitologia (MIP) na literatura científica nacional. Ci. Inf., Rio de Janeiro, 5(1/2) :43-69, 1976.

20. STEARNS, N. S. et alii. A core nursing library for practitioners. Amer. J. Nurs., New York, 70(4):818-23, Apr. 1970.

21. ...... \& RATCLIFF, W. W. An integrated health science core library for physicians, nurses and allied health practioners in community hospitals.

22. UNIVERSIDADE DE SÃO PAULO. EScola de Enfermagem. Programa de ensino. São Paulo, 1977.

23. ZOLTOWSKI, V. Les cycles de la creátion intelectuelle et artistique. In: L'ANNEE sociologique. Paris, Presses Universitaires de France, 1955, págs. 163-206. 